\title{
Effect Evaluation of Sterile Distilled Water in the Nursing Process of Patients with Abdominal Incision Endometriosis
}

\author{
LEI HAN, WEI NA ZHANG, MIN XU, ZHI QIN TONG AND YA JUAN PAN* \\ Department of Operation, Haian people's Hospital, No. 17, Zhongba Middle Road, Hai'an, Nantong, Jiangsu 226600, China
}

Han et al.: Evaluation of sterile distilled water in patients with abdominal incision endometriosis

\begin{abstract}
To explore the value of sterile distilled water in the care of patients with endometriosis in abdominal wall incision.120 patients who underwent cesarean section under combined lumbar and rigid anesthesia in our hospital from August 2017 to January 2018 were selected as study subjects, and all patients were treated surgically, and were randomly divided into three experimental groups according to their nursing treatment after surgery, control group I $(n=40)$ : using saline rinse; control group II $(n=40):$ using $0.5 \%$ povidone-iodine rinse; experimental Group $(n=40)$ : sterile distilled water rinses were used. Observation and comparison of patients' pain status before and after care, quality of life after care and satisfaction with care and the occurrence of complications. The pain and quality of life scores of the three groups of patients before nursing were not significantly different, and after nursing, the pain of patients in all groups was reduced, and the pain and quality of life of patients in the control II group and the test group were significantly reduced compared with the control I group $(p<0.05)$, and there was a significant difference in the quality of life comparison between the control II group and the experimental group (p<0.05), while the difference in pain was not statistically significant $(p>0.05)$. After care, the satisfaction of patients in the control II group and the test group was significantly higher $(p<0.05)$ compared to the control I group, and the test group had the highest satisfaction, but the difference between the control II group and the experimental group was not statistically significant $(\mathbf{p}>\mathbf{0 . 0 5})$. Compared with the control I group, patients in the control II and test groups had significantly lower complication rates, but the difference between the control II and experimental groups was not statistically significant $(p>0.05)$. The application of sterile distilled water rinsing in the care of patients with abdominal wall incision endometriosis can reduce the pain and alleviate the suffering of patients, while also effectively preventing complications and helping to shorten the recovery process.
\end{abstract}

Key words: Endometriosis, abdominal wall incision, sterile distilled water, care

Endometriosis (EMS) is a common gynecological disease in which the growth function of endometrial glands and stroma occurs in other parts of the uterus. Although the pathological process is benign, it has the ability to attack, implant and distant metastasis similar to malignant tumor cells, with an incidence rate of 8 $-15 \%{ }^{[1]}$. Abdominal incision endometriosis (AIEM), as akind of endometriosis, is a special part of endometriosis, the incidence is about $0.03 \%-1.73 \%{ }^{[2]}$. Cesarean section because of abdominal wall incision and uterine incision near parallel, close position, endometrial implantation opportunities. Therefore, AIEM secondary to cesarean section (about $90 \%$ ) is one of the main long-term complications after cesarean section ${ }^{[3]}$. At present, most studies have found that iatrogenic endometrial implantation caused by surgical procedures is a key factor in the occurrence of AIEM ${ }^{[4]}$. Therefore, standardized cesarean section and careful cleaning of incision are important preventive measures to prevent the occurrence of AIEM ${ }^{[5,6]}$. Studies have shown that incision irrigation can significantly reduce the number of endometrial debris on uterine incision, which is a simple and effective measure to prevent AIEM, and has been widely recognized and applied by the medical community ${ }^{[7]}$. At present, normal saline ${ }^{[8]}$ and $0.5 \%$ povidone iodine ${ }^{[9]}$ are commonly used to prevent AIEM. Although a large amount of normal saline washing can remove the fallen cell debris and prevent the occurrence of AIEM ${ }^{[10]}$. However, a large amount of washing can cause pressure on the incision and repeated friction, which will affect the healing of the wound. It can also make the bed sheet moist, bacteria breed and moderate 
amount of reduce body temperature probably by resetting the hypothalamus ${ }^{[11]}$. Sterile distilled water, a kind of low permeability liquid without any impurities and visible components, has been widely used to prevent tumor local spread and metastasis. Previous studies have found that the application of distilled water in breast cancer surgery is safe and effective, which is conducive to wound healing, but there are few reports on its application in AIEM $^{[12]}$. Therefore, this study intends to explore the application value of sterile distilled water in the nursing care of patients with abdominal incision endometriosis. A total of 120 patients with cesarean section under combined spinal epidural anesthesia from August 2017 to January 2018 were selected as the research objects. Inclusive criteria: lower segment cesarean section with transverse incision under combined spinal epidural anesthesia; primipara; no history of dysmenorrhea and endometriosis before pregnancy; no patients with basic diseases such as pregnancy induced hypertension and gestational diabetes mellitus. Exclusion criteria: patients with history of iodine allergy; patients with mental history or difficult to communicate with each other and unwilling to participate; those without complete cases and followup data. According to the principle of randomized controlled grouping, the patients were randomly divided into three groups: control group I $(n=40)$ : washed with normal saline; control group II $(\mathrm{n}=40)$ : washed with 0.5 $\%$ povidone iodine; experimental group $(n=40)$ : washed with sterile distilled water. Statistical analysis showed that there was no significant difference in age, course of disease, incision type and average weight among the three groups $(p>0.05)$, as shown in Table 1. All the three groups were operated by the same group of doctors, and the standardized operation of prevention of AIEM was strictly carried out during the operation: gauze was used to protect the incision area of abdominal wall after routine abdominal incision; amniotic fluid and blood were quickly sucked after uterine incision; hand contact was not allowed for wiping the gauze in uterine cavity; gloves, surgical instruments, suture thread, gauze and suction head were changed before closing the peritoneum. In the experimental group, the abdominal incision was washed with distilled water, and the specific steps were as follows: first, the surgical film was applied to the left edge of the incision, parallel to the longitudinal axis of the patient's body. The water bag is naturally drooping and knotting along the edge of the operating table to collect flushing fluid. Secondly, put the "s" hook under the incision side (both left and right sides, subject to the convenience of the operator), and press the side of the hook down slightly to make it at the lowest point. Pour $200 \mathrm{ml}$ of normal temperature flushing solution from top to bottom to completely submerge the surgical incision. Use the retractor to block the poured water at the incision for $5 \mathrm{~min}$. In control group I, abdominal incision was washed with normal saline. In control group II, $0.5 \%$ povidone iodine was used to wash abdominal incision, and the operation procedure was basically the same as that of the experimental group. The pain status of patients before and after nursing was compared. The visual analogue scale (VAS) was used to evaluate the pain status, which was divided into $0-10$ points, of which 0 points were painless and 10 points were severe pain. The quality of life of patients before and after nursing was compared. SF-36 scale was used to evaluate the quality of life, including physical function, emotional function, physical pain, social function and general health. The total score is 100 points, and the higher the score, the higher the quality of life. The patients' satisfaction with nursing work was compared before and after nursing. The form of anonymous questionnaire was used, including nursing attitude and nursing content. The full score was 30 points, 15 questions for each item, and the total score was more than 24 points. The incidence of complications, including pain, wound infection, tachycardia. Before nursing, all patients in the three groups had moderate pain. After nursing, the pain of all groups was reduced, and compared with control group I, the pain of control group II and experimental group was significantly reduced $(\mathrm{p}<0.05)$, but there was no significant difference between control group II and experimental group ( $>0.05$ ) as shown in fig. 1. There was no significant difference in the quality of life among the three groups. After nursing, the quality

TABLE 1: COMPARISON OF GENERAL CLINICAL DATA

\begin{tabular}{lccccc}
\hline Factors & Normal saline group & Distilled water group & $\mathbf{5 \% \text { povidone iodine group }}$ & $\mathbf{x}^{2}$ & $\mathrm{p}$ value \\
\hline Age $(\mathrm{y})$ & $32.1 \pm 2.3$ & $32.3 \pm 1.8$ & $32.2 \pm 2.5$ & 0.945 & 0.977 \\
Course of disease (mon) & $18.7 \pm 3.6$ & $19.4 \pm 3.1$ & $18.4 \pm 2.7$ & 1.055 & 0.905 \\
Type of incision & & & & & \\
Transverse incision & 17 & 19 & 20 & 0.968 & 0.940 \\
Longitudinal incision & 23 & 21 & 20 & & \\
weight & $52.99 \pm 2.23$ & $53.17 \pm 2.20$ & $53.05 \pm 2.40$ & 1.120 & 0.875 \\
\hline
\end{tabular}


of life of all groups of patients were improved, and compared with control group I, the pain of control group II and experimental group was significantly reduced $(\mathrm{p}<0.05)$, and the difference between control group II and experimental group was markedly significant $(\mathrm{p}<0.05)$, as shown in fig. 2. After nursing, the satisfaction of patients in the control group II and the experimental group was significantly higher than that in the control group I $(p<0.05)$, and the satisfaction of the experimental group was the highest, but there was no significant difference between the two groups $(\mathrm{p}>0.05)$, as shown in fig. 3. Compared with control group I, the incidence of complications in control group II and experimental group was significantly lower than that in control group, but there was no significant difference between control group II and experimental group ( $\mathrm{p}>0.05$ ), as shown in fig. 4. Pregnant women with a history of cesarean section, and with the following performance, should first think of abdominal wall endometriosis: typical menstrual cycle, abdominal wall incision scar pain, combined with painful nodules or masses, menstrual mass enlargement, pain aggravation, after menstrual mass reduction, pain relief or relief ${ }^{[13-15]}$. IEM, a relatively rare complication after cesarean section is secondary to 2-4 y after cesarean

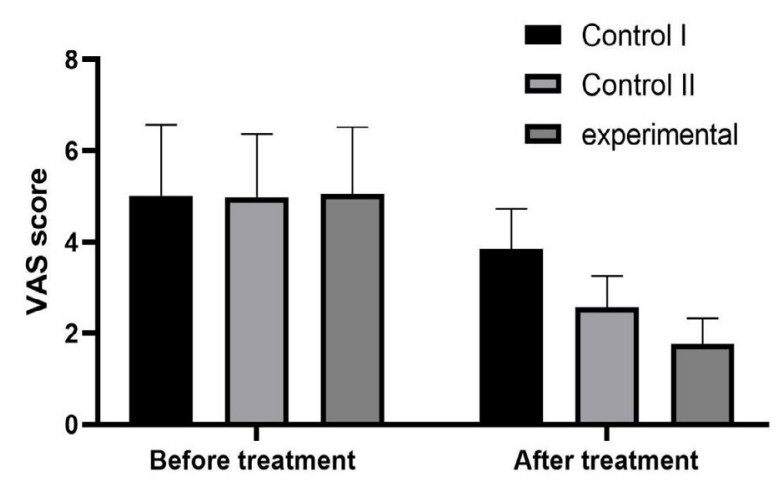

Fig. 1: Comparison of pain before and after treatment in three research groups

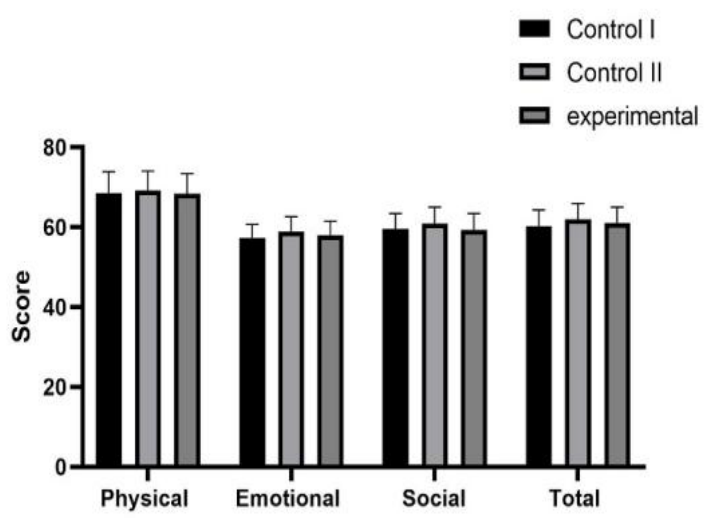

section. In recent $y$, the incidence rate of AIEM has also increased significantly due to the increase of cesarean section rate. Most scholars believe that iatrogenic endometrial implantation is the key factor of AIEM ${ }^{[16]}$. In the process of cesarean section, active endometrial cells are directly planted in the surgical wound, and eventually develop into scar endometriosis ${ }^{[17]}$. Ectopic to the incision endometrium tissue can occur periodic bleeding with the periodic changes of ovarian hormone, accompanied by fibrous tissue hyperplasia and adhesion formation, resulting in pain, mass, periodic abdominal incision mass enlargement and menstrual pain, which often brings pain to patients. Incision irrigation, as a simple and effective measure to prevent AIEM, can significantly reduce the number of endometrial debris on uterine incision, which has been widely recognized and applied by the medical community ${ }^{[9]}$. At present, normal saline and $0.5 \%$ povidone iodine are commonly used to prevent AIEM ${ }^{[11]}$. The normal saline has a basic property that can inhibit the bacterial growth, sometimes as bactericidal with the rise in its concentrations. The purpose of washing the wound surface with normal saline is to use the floating and fluidity of the liquid to remove the foreign bodies and falling tissue fragments on the surface of the incision, so that there is no free matter on the surface of the wound, so as to achieve the purpose of cleaning ${ }^{[12]}$. Studies have shown that a large number of normal saline washing can remove the fallen cell debris and prevent the occurrence of AIEM ${ }^{[13]}$. However, a large number of washing can cause pressure on the incision and repeated friction, which will affect the wound healing, and also make the bed sheet moist, bacteria breeding, and temperature reduction ${ }^{[14]}$. Povidone iodine is a kind of surfactant with strong adhesion. When it contacts the mucosa, it gradually decomposes and slowly releases active iodine, which destroys the protein and enzyme of the bacteria, and the metabolism of the offspring will be blocked and die ${ }^{[15,16]}$.

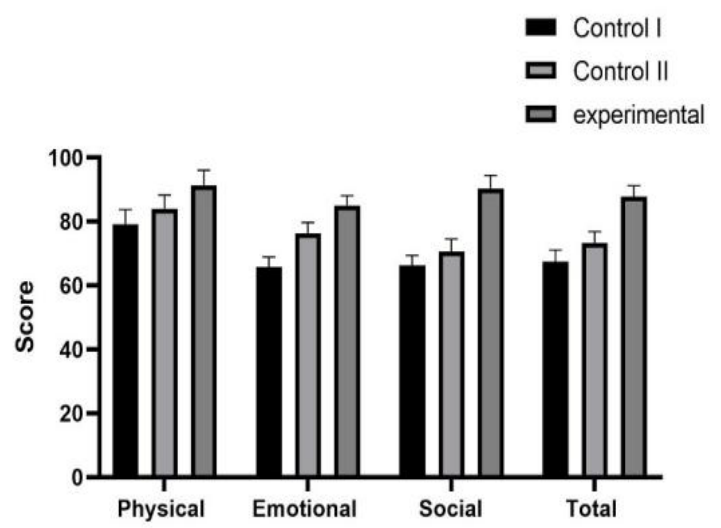

Fig. 2: Changes in quality of life of the three experimental groups before and after treatment 


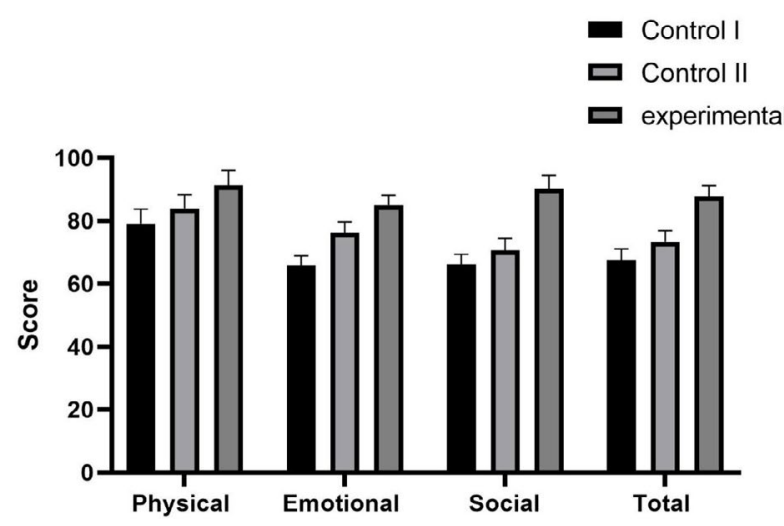

Fig. 3: Investigation on treatment satisfaction of three research groups

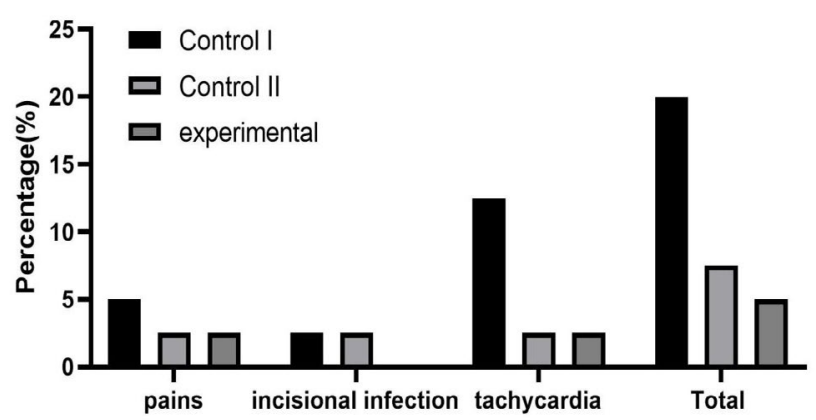

Fig. 4: Comparison of complications in the treatment of three research groups

Using this characteristic to make endometrial cells degenerate and necrosis prevent the occurrence of endometriosis. Balin et al. found that the proliferation activity of human skin fibroblasts cultured in vitro with different concentrations of povidone iodine decreased with the increase of povidone iodine concentration, and promoted cell apoptosis, suggesting that povidone iodine is toxic to human skin fibroblasts and may affect wound repair ${ }^{[17,18]}$. Chen Tao, et al. also found that repeated immersion and washing with $0.5 \%$ povidone iodine solution during the operation will form a chemical burn on the soft tissue and mucosa in the knee joint, causing inflammatory reaction of surrounding tissues, which is not conducive to wound healing ${ }^{[19]}$. Therefore, it is suggested that we should be careful when using povidone iodine for incision irrigation. Sterile distilled water, a kind of low permeability liquid without any impurities and visible components, has an osmotic pressure of about zero, while the cell osmotic pressure of normal human tissues is generally 280-310 osm. Therefore, there is a large difference in osmotic pressure between the two. If the cell is in a hypotonic environment, there will be a certain osmotic pressure difference between the inside and outside of the cell. At this time, the hypotonic solution can enter the cell through the cell membrane, causing direct swelling, dissolution and breakage of the cell and the number of damaged cells will continue to increase with the decrease of osmotic pressure ${ }^{[20]}$. Distilled water irrigation has been widely used in clinic to prevent local spread and metastasis of tumor. Studies have shown that the application of distilled water in breast cancer surgery is safe and effective, which is conducive to wound healing. In addition, the application of distilled water immersion washing can inactivate tumor cells and prevent infection through physical flushing ${ }^{[21]}$. In this study, the pain and quality of life of patients treated with sterile distilled water were significantly improved compared with the control group, suggesting that sterile distilled water can improve the quality of nursing in the operating room, and give patients safe and reliable treatment. Meanwhile, in terms of treatment satisfaction and psychological state evaluation, patients in the experimental group were significantly higher than the other two groups, which showed that sterile distilled water can give patients better treatment experience and enhance their confidence in treatment and rehabilitation. In addition, in the treatment of complications, the proportion of patients in the experimental group is the smallest and the degree is the lightest, which indicates that sterile distilled water can effectively and quickly control the side effects in the treatment process, reduce unnecessary medical disputes and the economic burden of patients. In conclusion, the application of sterile distilled water in the nursing care of patients with endometriosis of abdominal incision can reduce the pain and pain of patients, and effectively prevent complications, which is conducive to shorten the rehabilitation process.

\section{Acknowledgement:}

This work was supported by the foundation of Nantong Science and Technology Bureau Project (No. YYZ17054).

\section{Declaration of interest:}

The authors report no conflicts of interest.

\section{REFERENCES}

1. Bowes H, Jones G, Thompson J, Wood H, Hinchliff S, Ledger $\mathrm{W}$, et al. Understanding the impact of the treatment pathway upon the health-related quality of life of women with newly diagnosed endometrial cancer-A qualitative study. Eur J Oncol Nurs 2014;18:211-7.

2. Marsden NJ, Wilson-Jones N. Scar endometriosis: A rare skin lesion presenting to the plastic surgeon. J Plast Reconstr Aesthet Surg 2013;66:e111-3.

3. Leng J, Lang J, Guo L, Li H, Liu Z. Carcinosarcoma arising 
from atypical endometriosis in a cesarean section scar. Int $\mathrm{J}$ Gynecol Cancer 2006;169:432-5.

4. Kuhn PJ, Threlfall DR. Biosynthesis and metabolism of sesquiterpenoid phytoalexins and triterpenoids in potato cell suspension cultures. Phytochemistry 1988;27:133-50.

5. Gao Changyan, Ye Guoliu, Zhang Shuqin. 24 cases of abdominal wall incision after caesarean section.Care of patients with endometriosis. Med Adv 2013;3:162.

6. Hou XX, Yan QJ. Prevention of endometriosis during cesarean section with abdominal wall incision Influencing factors and nursing interventions for disorders of position. Chin Med J 2014;23:197-9.

7. Balleyguier C, Chapron C, Chopin N, Helenon O, Menu Y. Abdominal wall and surgical scar endometriosis: results of magnetic resonance imaging. Gynecol Obstet Invest 2003;55:220-4.

8. Liu LM, Gan QD, Guan JW. Prevention of abdominal wall incision during cesarean section Nursing interventions for endometriosis. Contemp Nurse 2012;16:97-8.

9. Chen J. Analysis of the effect of nursing intervention on laparoscopic endometriosis surgery patients. Psychological Monthly 2019;14:78-9.

10. Zhou ZW, Lin YQ. Endometriosis of the abdominal wall incision after cesarean section the preventive care measures. Zhejiang Med 2013;16:964-5.

11. Wang GX, Ren QZ, Jiang XL. Prevention of endometriosis with povidone-iodine in abdominal wall incision. $\mathrm{J}$ Tradit Chin Med 2013; 35:181-2.

12. Yu YJ, Sun SH, Geng SK. Study on the appropriate dosage of saline solution for wound rinsing in wound debridement surgery. Chinese Journal of Disability Medicine 2010;18:3-4.

13. Xiang Y. Report of 15 cases of abdominal wall incision for endometriosis. Chin J Obstet Gynecol 1991;26:303.
14. Ma YQ, Feng XX, Wang LW. Observation on the healing effect of subcutaneous irrigation on abdominal surgical incision in obstetrics and gynecology. Chin J Clin Res 2003;8:11974-5.

15. Ba JB, Liu YM, Yin M. Progress in the study of the physicochemical properties of povidone iodine and its disinfection applications. J Chin Disinfec 2009;26:548-50.

16. Liu YF, Zhao JM, Bai TC. Prevention of peritoneal implantation metastasis of gastric cancer by intraperitoneal lavage during povidone-iodine surgery. Chin J Mod General Surgery 2011;14:242-3.

17. Balin AK, Pratt L. Dilute povidone iodine solutions inhibit human skin fibroblast growth. Dermatol Surg 2002;28:210-4.

18. Yang GS, Zhang R, Feng YQ. Effect of different iodine preparations on the adhesion and proliferation of human skin fibroblasts. Chin J Mod Med 2010; 20:346-9.

19. Chen T, Shang XF, He R. Effect of iodophor flushing on incision and functional recovery after total knee replacement surgery. Clin Orthop 2011, 14(6): 697-699.

20. Guo GZ, Jia WJ. The use of tumor-free technique in radical breast cancer surgery. Chin General Nursing 2008, 6(10): 2792-2793.

21. Yi ZF, Zhu BR. The effect of warm distilled water for wound irrigation in breast cancer surgery. Chin Med Innov 2011, 10(30): 107-108.

This is an open access article distributed under the terms of the Creative Commons Attribution-NonCommercial-ShareAlike 3.0 License, which allows others to remix, tweak, and build upon the work non-commercially, as long as the author is credited and the new creations are licensed under the identical terms

This article was originally published in a special issue, "Biomedical Research in Clinical and Preclinical Pharmaceutics" Indian J Pharm Sci 2020:82(5)Spl issue7;82-86 